\title{
An Adolescent with Jaundice and Respiratory Distress from the Case Records of Kanchi Kamakoti CHILDS Trust Hospital
}

\author{
S Balasubramanian ${ }^{1}, \mathrm{~K}$ Dhanalakshmi ${ }^{2}$, Sumanth $\mathrm{A}^{3}$, Sanjay Deshpande ${ }^{4}$, Silky Agrawal ${ }^{5}$
}

Pediatric Infectious Disease (2019): 10.5005/jp-journals-10081-1110

Dr. Sanjay: A 15-year-old adolescent girl was admitted with a fever of 15 days duration, cough with respiratory distress for 7 days and icterus noticed for two days. She also had one episode of the seizure (GTCS), which lasted for 2 minutes on the day of admission. Before admission, she was treated outside as community-acquired pneumonia with broad-spectrum antibiotics (PiperacillinTazobactam) along with packed red blood cell (PRBC) transfusion.

\section{EXAMINATION FINDINGS}

On examination she was underweight (BMI-14), sick, pale and mildly icteric with respiratory distress and hypoxemia $\left(\mathrm{SpO}_{2} 84 \%\right)$. The liver was palpable $3 \mathrm{~cm}$ below the costal margin with a firm consistency. Examination of the cardiovascular system and the nervous system did not reveal abnormalities. There were scattered crackles in both lungs.

\section{INITIAL LABORATORY TEST}

Investigations revealed anemia with positive direct Coombs test (DCT), conjugated hyperbilirubinemia, transaminitis with low serum albumin and deranged coagulation profile (Table 1).

Dr. SBS: The clinical scenario is of an adolescent girl, with a relatively prolonged acute febrile illness and multisystem involvement

Table 1: Initial work up for PUO

\begin{tabular}{ll}
\hline Parameter & Value \\
\hline $\mathrm{Hb}$ & $9.4 \mathrm{~g} \%$ \\
Total count & $8900(\mathrm{~N}-61 \%, \mathrm{~L}-39 \%)$ \\
Platelets & 5.9 Lakhs \\
$\mathrm{DCT}$ & $2+$ \\
Malarial parasite (PS) & Negative \\
CRP & 50 \\
Renal function test (RFT) & Normal \\
Liver function test (LFT) & Bilirubin-5.7 mg/dL (DB-5.6), \\
& SGOT-990 \\
& SGPT 367 \\
& ALP 330 \\
& GGT-368 \\
& Albumin-2.6 \\
& PT-18.9/12 \\
& PTT-42/28 \\
& INR-1.67 \\
& Hypoechogenic liver \\
\hline
\end{tabular}

${ }^{1}$ Director and Head, ${ }^{2-4}$ Junior Consultant, ${ }^{4}$ Senior Clinical Fellow ${ }^{5}$ Junior Clinical Fellow

${ }^{1-5}$ Department of Pediatrics, Kanchi Kamakoti CHILDS Trust Hospital, Nungambakkam, Chennai, Tamil Nadu, India

Corresponding Author: S Balasubramanian, Director and Head, Department of Pediatrics, Kanchi Kamakoti CHILDS Trust Hospital, Nungambakkam, Chennai, Tamil Nadu, India, e-mail: sbsped@gmail. com

How to cite this article: Balasubramanian $S$, Dhanalakshmi $K$ Sumanth A, Deshpande S, Agrawal S. An Adolescent with Jaundice and Respiratory Distress from the Case Records of Kanchi Kamakoti CHILDS Trust Hospital. Pediatr Inf Dis 2019;1(1):39-41.

Source of support: Nil

Conflict of interest: None

(pulmonary, hematological, hepatic), which fits in the definition of PUO.

Differentials mainly consist of the three major groups of causes for PUO with severe pneumonia and clinical jaundice (Table 2).

Dr SBS: Dr Dhanalakshmi, is there a possibility of TB in this adolescent girl?

Dr K Dhanalakshmi: As you have rightly pointed out, any child presenting with fever of more than 2 weeks along with cough and respiratory distress, the possibility has to be kept in mind. The incidence of TB is more in two age groups (children $<2$ years and in the adolescent period). However, there is neither a history of contact with TB nor any significant illness in the past. Since there is simultaneous involvement of the liver and lungs, the possibility of viral infection/connective tissue disorder looks more likely. Malignancy looks unlikely because there is no lymphadenopathy or mass anywhere, mildly elevated platelets.

\begin{tabular}{ll}
\hline Etiology & Causes \\
\hline Infection & Viral (CMV,EBV, etc.) \\
& Atypical infections \\
& Bacterial-Burkholderia \\
& (meliodosis) \\
& Ricketssial-scrub \\
& Mycoplasma \\
& TB \\
& Systemic lupus erythematosus \\
(SLE), systemic onset juvenile \\
idiopathic arthritis (SOJIA) \\
Leukemia and lymphoma \\
\hline
\end{tabular}

(O) The Author(s). 2019 Open Access This article is distributed under the terms of the Creative Commons Attribution 4.0International License (https://creativecommons. org/licenses/by-nc/4.0/), which permits unrestricted use, distribution, and non-commercial reproduction in any medium, provided you give appropriate credit to the original author(s) and the source, provide a link to the Creative Commons license, and indicate if changes were made. The Creative Commons Public Domain Dedication waiver (http://creativecommons.org/publicdomain/zero/1.0/) applies to the data made available in this article, unless otherwise stated. 
In view of worsening respiratory distress and hypoxemia, she was shifted to PICU for respiratory support (CPAP) and was started on IV meropenem and doxycycline.

Dr. SBS: Dr. Sumanth, how do you interpret this X-ray and investigations?

Dr. Sumanth: The chest X-ray shows bilateral non-homogeneous infiltrates suggestive of ARDS. Since she was dependent on CPAP, CT chest was done which showed bilateral basal alveolar opacities and bilateral infiltrates (Fig. 1). We need to pursue further for an etiological diagnosis.

Dr. Sanjay: The rest of the investigations are available now (Table 3).

Over the past 4 days, she has not improved, her fever spikes are still persisting. Blood cultures have not shown any signals so far and currently, she is on meropenem and doxycycline.

Dr. SBS: I remember asking for an ophthalmology consult and a 2D-ECHO as part of the diagnostic workup for PUO.

Dr. Sanjay: The ophthalmologist has reported bilateral retinitis, which he feels that it may be infective or inflammatory in origin. ECHO does not reveal abnormalities.

Dr. SBS: At this stage, we have an adolescent girl with Interstitial pneumonia with ARDS with hepatic, hematological, hepatic and ophthalmic involvement and we need to rule out atypical and unusual infections or common ones with the unusual course. We can try to get the bug by doing a Broncho-alveolar lavage (BAL). Dr. Silky: BAL was done today. The following are the reports of BAL fluid: Gene-Xpert positive, rifampicin sensitive. Now we have an adolescent with microbiologically confirmed disseminated TB.

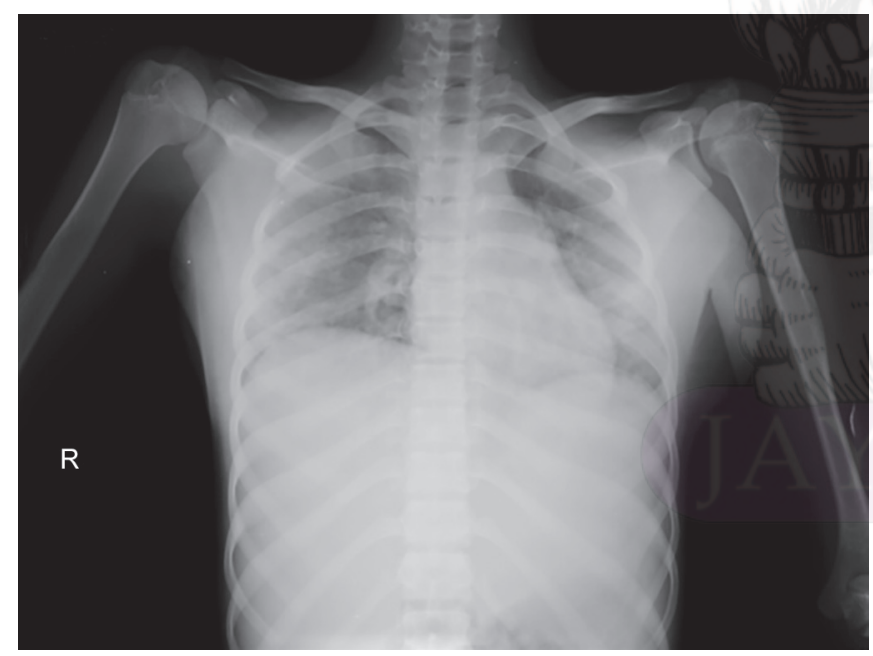

Fig. 1: Chest X-ray showing bilateral peribronchial infiltrates and the radiologist suggested the possibility of ARDS

Table 3: Serological and other tests

\begin{tabular}{ll}
\hline Diagnostic tests & Results \\
\hline Scrub Typhus IgM & Negative \\
Mycoplasma IgM & Negative \\
EBV VCA IgM & Negative \\
Viral hepatitis markers (Hep A & Negative \\
IgM, HBsAg, Hep E IgM), HIV & \\
ELFA & \\
ANA & Negative \\
Gastric lavage-AFB smear and & Negative \\
gene-expert & \\
\hline
\end{tabular}

Meanwhile, her respiratory distress worsened with impending respiratory failure, and she was intubated and ventilated and started on steroids for ARDS. What regimen of ATT should we start, keeping in mind the associated hepatitis?

Dr. KDhanalakshmi: The BAL fluid was sent for MGIT. Since there is significant transaminitis (more than 3 times the upper limit) and we will start on-levofloxacin, amikacin, ethambutol (nonhepatotoxic regime) and the steroids can be continued.

After starting ATT, her fever spikes were coming down, but jaundice was worsening even though we are giving nonhepatotoxic drugs. Is there a possibility of autoimmune hepatitis component as well, Sir?

Dr. SBS: Since she is an adolescent girl and her liver parameters are worsening in spite of nonhepatotoxic regimen, we need to rule out co-existing autoimmune hepatitis. Hence we will workup for the same along with a liver biopsy

Dr. Sumanth: The workup for autoimmune hepatitis is as followsLKM antibodies and ANA are negative and a repeat USG showed no new findings. The liver biopsy report is that of a portal expansion with multiple epitheloid granulomas, portal lymphocytic inflammation with spotty necrosis, few foamy histiocytes, AFB stain negative-granulomatous hepatitis (possibly infective). With the above investigations, autoimmune hepatitis is unlikely and the liver involvement is a probably part of the disseminated TB affecting the liver.

Dr. Sanjay: Can we continue the same regimen for TB?

Dr. SBS: Yes, we will continue the same regimen along with steroids

Meanwhile, her fever settled, and jaundice was decreasing, and her appetite improved. She was discharged on ATT with tapering doses of steroids.

Dr. Silky: The same girl is now re-admitted (after a week) with high-grade fever and generalized maculopapular rash. However, she has improved clinically and radiologically now presenting with only fever for and maculopapular rash for 2 days. The workup shows a normal CBC and viral serologies for EBV/CMV IgM, are negative. However, a repeat ophthalmological consult revealed the persistence of choroidal tubercles.

Dr. Dhanalakshmi: Since there is evidence of ocular involvement of TB, we will restart steroids (prednisolone $2 \mathrm{mg} / \mathrm{kg} / \mathrm{day}$ ) and continue ATT. If her liver parameters improve, we can reintroduce the first line ATT after a period of 3-4 weeks.

Dr. SBS: Tuberculous involvement of the liver, as a part of disseminated tuberculosis, is seen in up to $50-80 \%$ of cases. ${ }^{1}$ Common symptoms include: ${ }^{2}$

- Hepatomegaly (80\%)

- Fever (67\%)

- Respiratory symptoms (66\%)

- Abdominal pain and weight loss $(60 \%)$

- Splenomegaly $(25-55 \%)^{3}$

- $\quad$ Ascites and jaundice (20\%).

Hepatic TB can be classified into five clinical-pathological presentations: $^{4}$

1. Miliary TB

2. Granulomatous TB hepatitis

3. Nodular TB

4. Ductal TB

5. Nodal TB

Jaundice as a symptom of hepatobiliary TB was observed in $20-35 \%^{3}$ of patients in a study. The pathogenesis of jaundice in hepatobiliary and the presence of jaundice suggest biliary involvement, and the biochemical profile may simulate extrahepatic biliary obstruction. Published studies point out that jaundice or 
biliary stasis is due either to porta hepatis nodes causing biliary compression or to pericholangitis or possibly by direct involvement of biliary epithelium or the rupture of a tuberculous granuloma into the bile ducts. ${ }^{4,5}$ Diagnosis of hepatic and intra-abdominal TB can be challenging due to variable symptomatology and therefore requires a high level of suspicion. ${ }^{4}$ Frequent symptoms include abdominal pain, weight loss, weakness, abdominal distension, mass, diarrhea, and pyrexia. Biochemical markers associated with an infective process such as white cell count and CRP are often raised. Hepatic TB is often associated with deranged liver function tests (LFTs). The LFTs can reflect the site of hepatic TB, for example, TB affecting the liver parenchyma will be reflected in elevated transaminases as compared to alkaline phosphatase, and the contrary is true of extrahepatic TB affecting the porta or ducts where the alkaline phosphatase will show a proportionally greater rise as compared to transaminases. Extrahepatic TB will also cause an earlier rise in bilirubin as compared to parenchymal disease due to obstruction. Progression to liver failure will be evident in altered bilirubin, INR and albumin. Imaging plays a key role in the identification of hepatic and intra-abdominal TB. Ultrasound can facilitate the diagnosis of intra-abdominal and hepatic TB, as it is not only advantageous at imaging the liver but can also identify lymphadenopathy and ascites, which could be sampled for further analysis. CT is fast becoming the first-line imaging modality for abdominal symptoms and has been shown to be more sensitive in diagnosing TB than ultrasound. The diagnosis of TB even after successfully removing tissue can sometimes remain difficult. Microscopy, culture, histology, and CBNAAT are all important in achieving a diagnosis. In this adolescent, there was a primary hepatic tuberculous parenchymal disease as suggested by the granuloma and conjugated hyperbilirubinemia with transaminitis without elevation of SAP or Gamma GT. ${ }^{4}$

\section{Treatment}

Antitubercular therapy (ATT) is the cornerstone of treatment and duration of DSTB (drug-sensitive TB) is 9 months-12 months, sometimes extending up 15-18 months (RNTCP 2019).

Surgical interventions are needed in select circumstances. Up to $20-40 \%$ of patients with abdominal TB will present with an acute abdomen and require emergency surgery. Surgical intervention is as high as $85 \%$ in populations with a high incidence of TB, which is in contrast to developed nations where there is often a low incidence of TB and the proportion of children requiring surgery was $55 \%$. Expertise in pediatric $\mathrm{CT}$ and ultrasound-guided biopsy can reduce the need for surgery in the pediatric population. ${ }^{4}$

\section{Steroids in TB}

The role of adjunctive steroids in abdominal TB is not well established. Previous guidelines mention the use of steroids in TBM and pericarditis as definitive indications. ${ }^{6}$

However, the current RNTCP guidelines mention (RNTCP 2019) mention newer indications of the use of steroids in TB such as
Addison's disease, Miliary TB with alveolo-capillary block and TB uveitis. They may be used in endobronchial tuberculosis, bronchial compression, mediastinal compression syndrome, pleurisy with severe distress, laryngeal TB, TB IRIS and miliary disease with alveolocapillary block. ${ }^{7}$

DrSBS: Her LFT 2 weeks later returned to normal and she had started gaining weight. Regular first-line regimen for TB (HRZE) as per RNTCP protocol was gradually introduced which was well tolerated. It is 3 months since we started drugs for TB and her ocular lesions have regressed. Her BAL cultures for TB were negative.

TB of the liver and abdomen remains uncommon. A high level of suspicion is required to diagnose hepatic and intra-abdominal TB as it presents with nonspecific symptoms. Medical therapy remains the mainstay of treatment, but surgery still plays an important role in the diagnosis and the management of complications such as obstructive jaundice or hepatic abscess.

In retrospect, this adolescent girl had disseminated TB with granulomatous hepatitis and recovered with ATT and steroids.

\section{Highlights}

- TB still has a high prevalence and should always be considered in differentials while approaching a case of PUO regardless of age.

- While evaluating a case of PUO it is of importance to get an ophthalmological evaluation.

- Every effort must be made to get the microbiological diagnosis when the differential includes infective etiology especially.

- TB uveitis management involves both ATT and steroids.

- Even in the presence of jaundice, TB has to be considered in the differential diagnosis of PUO.

- Hepatic TB can present as PUO, jaundice or even as abscess.

\section{References}

1. Patel N, Amarapurkar A, Amarapurkar D. Hepatobiliary tuberculosis in western India. Indian Journal of Pathology and Microbiology. 2008;51(2):175.

2. Hickey A, Gounder L, Moosa M, Drain P. A systematic review of hepatictuberculosis with considerations in human immunodeficiency virus co-infection. BMC Infectious Diseases. 2015;15(1).

3. Hepatobiliary tuberculosis Poras Chaudhary Dr Ram Manohar Lohia Hospital (Dr RMLH and PGIMER), New Delhi, India Annals of Gastroenterology (2014;27:207-211

4. Evans R, Mourad M, Dvorkin L, et al. Hepatic and intra-abdominal tuberculosis: 2016 Update. Current Infectious Disease Reports 2016;18(12).

5. Kok K, Yapp S. Tuberculosis of the Bile Duct. Journal of Clinical Gastroenterology.1999;29(2):161-164.

6. Singh SK, Tiwari KK. Use of corticosteroids in tuberculosis. J Assoc Chest Physicians 2017;5:70-75.

7. RNTCP updated Pediatric TB guidelines 2019 Developed by revised national tuberculosis control program and Indian academy of pediatrics. 